\title{
CURRÍCULO OCULTO EN LA INVESTIGACIÓN FORMATIVA DEL PROGRAMA DE LICENCIATURA EN EDUCACIÓN FÍSICA
}

\author{
HIDDEN CURRICULUM IN FORMATIVE RESEARCH IN THE \\ BACHELOR'S DEGREE IN PHYSICAL EDUCATION \\ CURRÍCULO OCULTO NA PESQUISA FORMATIVA DO PROGRAMA
DE BACHARELADO EM EDUCAÇÃO FÍSICA
}

Juan David Paz Benavides ${ }^{1}$

Resumen

Este artículo de investigación pretende interpretar cómo influye el currículo oculto en la investigación formativa desarrollada en el Programa de Licenciatura en Educación Física de la Institución Universitaria Cesmag y la percepción que la comunidad académica tiene sobre él. Los resultados muestran que el currículo oculto construye actitudes, habilidades y competencias investigativas en los estudiantes, producto de la interacción con sus pares estudiantiles y docentes. Asimismo, se resalta la importancia de la experiencia investigativa en docentes que orientan, asesoran y evalúan los procesos investigativos desarrollados por los estudiantes del programa. También se evidencia que las actitudes conductuales negativas de los docentes son producto de una baja producción investigativa, y que de manera inconsciente se transmiten al estudiantado y generan relaciones académicas negativas, lo cual dificulta la construcción de competencias específicas de este campo.

Palabras clave: actitudes investigativas; competencias investigativas; currículo; investigación formativa

\section{Abstract}

This research article attempts to interpret how the hidden curriculum influences the formative research of the Bachelor's Degree in Physical Education of the Institución Universitaria Cesmag, and the perception that the academic community has about it. The results show that the hidden curriculum builds attitudes, skills and research competences in students, as a result of their interaction with their peers and teachers. Likewise, they highlight the importance of the research experience in teachers who guide, assess and evaluate the research processes developed by the students of the program. It is also evident that the teachers' negative behavioral attitudes are the product of a low research production. They are unconsciously transmitted to students and generate negative academic relationships, which makes the construction of specific competences in this field difficult.

Keywords: Curriculum; research attitudes; research competences; formative research

1 Magíster en Docencia Universitaria. Licenciado en Educación Física. Docente tiempo completo del Programa de Licenciatura en Educación Física en la Institución Universitaria Cesmag. Correo electrónico: jdpaz@iucesmag.edu.co. 


\section{Resumo}

Este artigo tem como objetivo interpretar como o currículo oculto influência na pesquisa formativa desenvolvida no Programa de Bacharelado em Educação Física da Instituição Universitária de Cesmag e na percepção que a comunidade acadêmica tem sobre ele. Os resultados mostram que o currículo oculto constrói atitudes, habilidades e habilidades investigativas nos alunos, como resultado da interação com seus colegas e professores. Da mesma forma, destaca-se a importância da experiência de pesquisa em professores que orientam, aconselham e avaliam os processos de pesquisa desenvolvidos pelos alunos do programa. Também é evidente que as atitudes negativas comportamentais dos professores são o resultado da baixa produção de pesquisa, e que elas são transmitidas inconscientemente aos alunos e geram relações acadêmicas negativas, o que dificulta a construção de competências específicas neste campo.

Palavras chave: Atitudes investigativas; competências investigativas; currículo; pesquisa formativa

Fecha de recepción: 22 de marzo de 2018

Fecha de aprobación: 10 de septiembre de 2018

Para citar este artículo:

Paz, J. (2019). Currículo oculto en la investigación formativa del Programa de Licenciatura en Educación Física. Lúdica Pedagógica, 28, 9-19. 


\section{INTRODUCCIÓN}

En este artículo se evidencia cómo el currículo oculto del Programa de Licenciatura en Educación Física de la Institución Universitaria Cesmag influye en el desarrollo de los procesos de investigación formativa realizados por los estudiantes-maestros. Por esta razón, es necesario conceptualizar sobre investigación formativa, currículo y currículo oculto.

\section{Investigación formativa}

La investigación formativa se considera una estrategia de gran importancia en la formación de los futuros profesionales. A través de esta, el estudiante relaciona los conocimientos construidos en los diferentes espacios académicos para aplicarlos en situaciones de mayor relevancia y generar soluciones a problemas propios de su quehacer profesional. Además, en el caso del Programa de Licenciatura en Educación Física, este campo se relaciona con la práctica pedagógica y permite a los educandos tener contacto directo con los diferentes contextos laborales.

La investigación formativa en el Programa de Licenciatura en Educación Física de la Institución Universitaria Cesmag se promueve e implementa a partir de los espacios académicos de Seminario de Investigación Propuesta de Investigación, Seminario de Investigación Proyecto de Investigación, Seminario de Investigación Sistematización Inicial y Seminario de Investigación Sistematización Final, en los cuales los estudiantes deben construir su trabajo de grado para optar al título profesional. Asimismo, el programa cuenta con el semillero de investigación Ambi Wiwa, el cual pretende motivar y estimular el desarrollo de actividades investigativas a través de capacitaciones, producción investigativa e intelectual, y participación de sus integrantes en eventos de índole académica. De igual manera, la estrategia interestructurante denominada Proyecto de Ambientes Académicos Formativos se trabaja en el tiempo independiente de los estudiantes a través de la plataforma Moodle, partiendo de un proceso comprensivo de la realidad desde los saberes específicos y tendiente al fomento de la investigación formativa.

Miyahira (2009), asume que desarrollar continuamente la investigación formativa permite a los estudiantes generar o mejorar sus capacidades de análisis, síntesis, interpretación de información y búsqueda de problemas no resueltos. Para los licenciados en Educación Física las capacidades de observación, descripción y comparación son fundamentales para ejercer su labor docente en los diferentes contextos educativos, ya que les permiten diagnosticar las dificultades que presentan sus estudiantes para el aprendizaje y ofrecerles alternativas de mejoramiento desde la disciplina.

Banguero (2008) sugiere que la investigación formativa proporciona una mirada multidimensional de los problemas, superando el reduccionismo en la comprensión de los fenómenos sociales, culturales y humanos; esto da como resultado la organización de conocimiento de carácter interdisciplinario. A través de un proceso investigativo es posible que los estudiantes del Programa de Licenciatura en Educación Física se acerquen a la construcción de saberes disciplinares, que permitan la transformación de los contextos en los cuales realizan su intervención pedagógica. Por lo anterior, la investigación formativa se convierte en un pilar fundamental de las instituciones de educación superior, debido a que contribuye al desarrollo de competencias que permitirán al futuro licenciado abordar con eficiencia su quehacer pedagógico en la sociedad. Así lo afirman Núñez y Vega (2011): “Investigar y promover la investigación es un punto nodal de la formación académica profesional, articulado en esta línea en la estructura curricular y el perfil profesional, como fines de la universidad" (p. 1).

Parra (2004) señala que la investigación formativa tiene como finalidad pedagógica mejorar los procesos de enseñanza-aprendizaje y se desarrolla dentro de un marco curricular establecido. La enseñanza a través de la investigación y la docencia investigativa es una estrategia pedagógica para el desarrollo del currículo.

\section{Currículo}

En la actualidad, el currículo no es exclusivamente un programa o una serie de contenidos educativos, es un proceso institucional en continua construcción que se incorpora en los salones, influye sobre los estudiantes y responde con pertinencia a las necesidades del entorno. Avendaño y Parada (2013), afirman que el currículo debe tener en cuenta el sistema sociocultural y político, para permitir que el direccionamiento de las instituciones sea adecuado y coherente 
con las necesidades de los sujetos que forman parte de este proceso pedagógico. El currículo debe ser un proceso de construcción interestructurante entre las instituciones educativas, la sociedad, los estudiantes y los docentes, con la finalidad de proporcionar a la comunidad individuos demócratas, autónomos y responsables.

Según Sánchez y Meza (2007), en el nuevo milenio los currículos de las universidades han reorientado su planificación, con el fin de desarrollar en los estudiantes las competencias necesarias para desempeñarse en sociedad y proporcionar herramientas para la construcción de conocimientos, habilidades $\mathrm{y}$ actitudes que garanticen un correcto accionar en el ámbito laboral. En este sentido, el currículo no debe limitarse a formar a los individuos solo con conocimientos teóricos, también debe hacerlo con habilidades prácticas y actitudes. Malagón (2008) conceptualiza el currículo como una práctica pedagógica que reconoce la construcción cooperativa entre individuos e instituciones educativas; en la educación superior permite encontrar la relación que existe entre la institución y el contexto, y facilita el aprendizaje de nuevos conocimientos construidos en contextos prácticos.

Eisner (1979), define el currículo educativo como "una serie de eventos planeados cuya intencionalidad es lograr consecuencias educacionales para uno o más estudiantes" (p. 39). Este autor considera que hay tres tipos de currículo: explícito, oculto y nulo. Para la construcción del presente artículo de investigación se profundizará sobre currículo oculto.

\section{Currículo oculto}

El currículo oculto es todo lo que no está estipulado en los planes de estudios o en la normativa del sistema institucional. Es de gran importancia en la investigación formativa, debido a que a través de él se pueden desarrollar actitudes en los estudiantes frente a este campo, por medio de las relaciones interpersonales y situaciones cotidianas.

Kirk (1990) afirma que el currículo oculto comprende valores, actitudes e ideologías que se transmiten a los estudiantes de manera voluntaria o involuntaria, como resultado de la puesta en práctica del currículo explícito. Carrillo (2009) considera que los conocimientos, destrezas, valores y actitudes que se construyen en los estudiantes son producto de todas las interacciones que se dan a diario en los contextos educativos, pero que generalmente no se encuentran explícitas en los documentos institucionales.

Este tipo de currículo muestra que las diferentes actividades desarrolladas en la institución traen resultados en la construcción de competencias investigativas del estudiantado. Díaz (2005) establece que el currículo oculto y sus aprendizajes están estrechamente relacionados con las competencias; además, este mantiene una cercana relación con el aprendizaje de valores en los educandos. Estos aprendizajes son producto de las relaciones que surgen entre los estudiantes y los demás estamentos que conforman la comunidad educativa.

En la cotidianidad de los centros educativos se encuentran actitudes que oscilan entre dos extremos. Magendzo, Abraham y Dueñas (1993) aseguran que estas son: actitudes positivas y negativas del profesor; y actitudes positivas y negativas entre estudiantes. Las actitudes negativas se caracterizan por juzgar y señalar constantemente el accionar del estudiante; las actitudes positivas, en cambio, valoran las virtudes del educando y contribuyen a mejorar sus debilidades; además, toda la comunidad académica se interesa y motiva el proceso formativo del estudiantado.

Asimismo, el currículo oculto presenta una clara incidencia de factores ajenos a la institución; tal como lo afirma Rodríguez (2012), este currículo cuenta con algunos condicionantes externos, como las continuas transformaciones de la educación, el desarrollo de nuevas tecnologías y el progreso social. Los elementos mencionados condicionan aspectos como la modernización, la actualización y la pertinencia curricular; por lo tanto, también generan transformaciones en el currículo oculto.

\section{METODOLOGÍA}

Este artículo es resultado de una investigación etnográfica de corte cualitativo y enfoque hermenéutico, El objetivo general de este estudio fue comprender la investigación formativa de los estudiantes del Programa de Licenciatura en Educación Física. El objetivo específico sobre el cual se desarrolla este artículo es describir la investigación formativa que propone el currículo del Programa de Licenciatura en Educación Física. 
La unidad de trabajo correspondió a los cuatro docentes con título de magíster que orientan el espacio académico de Seminario de Investigación, el director de programa y 33 estudiantes del Programa de Licenciatura en Educación Física de la Institución Universitaria Cesmag.

Las técnicas de recolección de información utilizadas fueron entrevista en profundidad y grupo focal. La entrevista en profundidad permite obtener datos importantes, resultado de un proceso planificado y continuo. Se debe evitar que las sesiones de entrevista sean demasiado largas, ya que esto genera cansancio y desinterés en el entrevistado; es posible aplicar esta técnica varias veces en una misma población, hasta que el investigador considere que ha recabado suficiente información (Robles, 2011). La entrevista en profundidad fue aplicada a los cuatro profesores que orientan el espacio académico de Seminario de Investigación y al director del Programa de Licenciatura en Educación Física. Para la ejecución de la técnica se contó con un tiempo de 35 minutos y se utilizó un guion de entrevista, compuesto por 7 preguntas sobre currículo oculto, que se relacionaban con las actitudes, las relaciones académicas y el contexto universitario.

La otra técnica utilizada fue el grupo focal, que se constituye en un espacio de participación para captar ideas y vivencias de los individuos. En ellos se presentan expectativas, explicaciones y soluciones hacia el tema propuesto por el investigador; es una técnica de recolección de información colectivista, debido a que se centra en la variedad de actitudes, experiencias y creencias de los participantes (Hamui y Ruiz, 2013). En este estudio se realizó un grupo focal en cada uno de los semestres que cursaban un espacio académico de Seminario de Investigación; en total participaron 33 estudiantes del programa. En la tabla 1 se muestra la distribución de cada grupo focal.

Tabla 1. Distribución de grupos focales

\begin{tabular}{|c|c|c|l|}
\hline Grupo focal & Número de estudiantes & Semestre & \multicolumn{1}{|c|}{ Espacio académico } \\
\hline 1 & 9 & $7-0$ & Seminario Propuesta de Investigación \\
\hline 2 & 8 & $8-0$ & Seminario Proyecto de Investigación \\
\hline 3 & 6 & 9.0 & Seminario Sistematización Inicial \\
\hline 4 & 10 & 10.0 & Seminario Sistematización Final \\
\hline
\end{tabular}

Fuente: elaboración propia.

Para la ejecución de esta técnica se contó con un tiempo de 50 minutos y se utilizó un guion de grupo focal, que contenía 7 preguntas sobre currículo oculto, las cuales estaban relacionadas con las actitudes, las relaciones académicas y el contexto universitario.

Antes de implementar la entrevista en profundidad y el grupo focal, fue necesaria la validación por juicio de expertos. Según Escobar y Cuervo (2008), este tipo de validación es una opinión de personas con conocimiento en el tema, quienes hacen aportes y evalúan los instrumentos de recolección de información. En esta investigación se contó con la participación de tres expertos con experiencia en investigación formativa en universidades de San Juan de Pasto.

\section{RESULTADOS}

\section{Actitudes docentes hacia la investigación formativa}

La comunidad académica del Programa de Licenciatura en Educación Física reconoce que mayoritariamente existen actitudes positivas de docentes frente a la investigación formativa, siendo el respeto, la motivación y el interés las actitudes que mencionaron las tres fuentes. Docentes y directivos concuerdan en que una actitud importante en los profesores es la satisfacción, entendida como el gusto por desarrollar procesos investigativos; esto hará que los estudiantes sientan interés por participar en ellos.

Por otra parte, docentes y estudiantes coinciden en que la responsabilidad y la disposición son nece- 
sarias para desarrollar investigación formativa. La responsabilidad se define como la disciplina y la organización en el proyecto investigativo. En cuanto a la disposición, esta se cataloga como aquella actitud en la cual el docente manifiesta toda su suficiencia en investigación, con el fin de aportar y colaborar en la construcción de competencias investigativas en el estudiantado.

Asimismo, la población consultada afirma que el perfil del docente que orienta la investigación es de suma importancia; por esta razón, el profesor de Seminario de Investigación debe tener experiencia investigativa, creatividad y ser capaz de contextualizar la investigación. En el programa, estas tres características son evidentes en los docentes que imparten estos espacios académicos.

Se debe mencionar que tres de los cuatro docentes de Seminario de Investigación no son licenciados en Educación Física, esto proporciona a los proyectos estudiantiles un punto de vista diferente al de la disciplina. La población consultada ve como un elemento positivo la interdisciplinariedad con la que cuenta el Programa de Licenciatura en Educación Física; consideran que los docentes realizan aportes al proceso investigativo desde diferentes puntos de vista, lo cual enriquece la construcción de los proyectos investigativos y la formación del estudiantado en actitudes positivas.

Se resalta que los educandos afirman que los docentes de Seminario de Investigación no tienen actitudes negativas frente a este campo. Por el contrario, en los demás profesores del programa algunos estudiantes identifican las siguientes actitudes negativas: indiferencia, desmotivación y desinterés. El estudiantado siente que los docentes no demuestran interés por la investigación formativa, no motivan el desarrollo de investigaciones innovadoras en el programa y, finalmente, algunos no realizan el acompañamiento adecuado a los procesos investigativos a través de las asesorías. Algunos jurados tampoco incentivan la construcción de proyectos investigativos innovadores, ya que los proyectos novedosos los obligarían a capacitarse en temas que desconocen.

\section{Actitudes estudiantiles hacia la}

investigación formativa

La actitud que prevalece en los estudiantes del programa es el interés, para esto es fundamental que elijan un tema de investigación de su agrado. En el programa también se evidencian otras actitudes positivas, como la motivación, la satisfacción, el compromiso y el sentido de pertenencia.

Según las fuentes consultadas, la motivación en los estudiantes determina en gran medida el éxito de los procesos de aprendizaje. Por otra parte, la satisfacción frente a la investigación se evidencia en los estudiantes cuando solucionan problemáticas del contexto desde la disciplina de la educación física. El compromiso y el sentido de pertenencia se evidencian en el vínculo que establece el estudiantado con la población en la cual realiza su intervención pedagógica e investigación.

Las actitudes positivas de los estudiantes frente a la investigación formativa aumentan significativamente a partir del séptimo semestre, cuando los futuros licenciados cuentan con el correcto acompañamiento de docentes investigadores en los espacios académicos de Seminario de Investigación, quienes promueven las actitudes adecuadas hacia este campo. En lo que respecta al desempeño en contextos reales, todas las fuentes de información concuerdan en que a través de las actitudes positivas de los estudiantes hacia la investigación es posible mejorar su desempeño en su práctica pedagógica.

Por el contrario, algunos estudiantes no consideran que la investigación formativa sea relevante para la profesión docente, y reconocen en ellos actitudes de pereza, aburrimiento y desinterés. De igual manera, existen actitudes negativas en los grupos de trabajo del proyecto de grado, como poca comunicación, poca cooperación e irresponsabilidad.

El Programa de Licenciatura en Educación Física aplica la estrategia metodológica del Proyecto de Ambientes Académicos Formativos para desarrollar competencias investigativas. Sin embargo, los estudiantes aseguran que esta estrategia no logra desarrollar habilidades de escritura, lectura o despertar su interés por la investigación formativa. También se debe mencionar que los espacios académicos del campo investigativo en el ciclo de fundamentación generan actitudes negativas frente a la investigación.

\section{Relaciones académicas}

La comunidad académica señala que las características más notables de las relaciones académicas entre 
docentes y estudiantes son la confianza, la responsabilidad y la disposición. La confianza es aquella actitud en la cual el estudiante entiende y acepta los aportes realizados por los profesores; los estudiantes demuestran confianza cuando conocen la cantidad y calidad de conocimiento con el que cuenta el docente para orientar los procesos investigativos. La responsabilidad se define como un proceso recíproco necesario para avanzar en el proceso investigativo. Por último, la disposición es aquella actitud en la cual tanto docentes como estudiantes evidencian el interés por desarrollar procesos investigativos.

En contraparte, en el Programa de Licenciatura en Educación Física también existen relaciones académicas negativas, que se dan entre estudiantes y jurado de trabajo de grado, y entre estudiantes y docentes que no imparten catedra de espacios académicos del campo investigativo. Los estudiantes sugieren a los evaluadores de los proyectos ser más coherentes entre pares y así evitar dificultades en los procesos de investigación formativa que adelantan.

Generalmente los estudiantes perciben las sugerencias de los evaluadores como obstáculos que retrasan el proyecto investigativo y no como elementos que permitan mejorar la construcción de su trabajo de grado, debido a que algunos docentes del programa no están suficientemente formados en investigación, lo cual infiere de manera negativa sobre los educandos.

\section{DISCUSIÓN}

\section{Actitudes docentes hacia la investigación formativa}

La comunidad educativa participante en el proceso de recolección de información reconoce que los docentes de Seminario de Investigación tienen actitudes positivas frente a esta área. Cervantes, Cappello y Castro (2009) aseguran que las actitudes tienen una influencia positiva o negativa en nuestra vida cotidiana casi imperceptible; en actividades como la docencia, las actitudes deben ser un permanente objeto de estudio, ya que las actitudes positivas para la enseñanza contribuyen al mejor desempeño de profesores y estudiantes. El respeto en el programa se describe como la actitud más importante que presentan los docentes; Hildebrand (2004) considera que esta es la virtud fundamental que permite la construcción de todas las actitudes positivas.
Junto con el respeto, otras actitudes de gran importancia son la motivación, la satisfacción y la disposición. Aldana y Joya (2011) sugieren que las anteriores son actitudes cognoscitivas, en las cuales se representan los conocimientos teórico, procedimental y actitudinal que posee el docente en materia de investigación. La motivación es la actitud que debe predominar en los procesos de investigación formativa; la satisfacción se evidencia a través de los sentimientos de agrado hacia la investigación.

Por último, la disposición es la actitud que demuestra el profesor para poner al servicio del estudiantado todo su conocimiento en materia de investigación, con el objetivo de aportar al proceso de formación del educando (Aldana y Joya, 2011).

Además de la actitud positiva, el perfil del docente que orienta la investigación es de suma importancia; por esta razón, el profesor de Seminario de Investigación debe tener perfil de investigador. Pirela y Prieto (2006) coinciden en que

Los docentes como investigadores deben desarrollar las competencias, con énfasis en el dominio de los términos, procesos y teorías del campo de la investigación, fundamentadas en el razonamiento científico, que le permita abordar de manera crítica la realidad, construir mapas cognoscitivos y valorativos que expliquen la misma, utilizar la capacidad de análisis y síntesis, juicio crítico, motivación al logro, entre otros, para generar de esta forma nuevos conocimientos. (p. 161).

El profesor de Seminario de Investigación debe ser una persona con experiencia investigativa, creativa y capaz de contextualizar la investigación a la disciplina de la educación física. Para Hernández (2009) el docente investigador puede construir conocimiento en sus estudiantes, de manera que desarrollen competencias específicas de cada profesión, que les permitan hacer frente a las situaciones que encontrarán en los diferentes contextos de su vida profesional. En cuanto a la creatividad, esta evita que la investigación se convierta en un proceso técnico y promueve actitudes positivas en los estudiantes frente a los procesos investigativos (Campos, 2003).

Por otra parte, toda la comunidad académica percibe como una fortaleza la interdisciplinariedad con la que cuenta el programa, gracias a la experiencia investigativa y la creatividad con la de los docen- 
tes que orientan los espacios académicos del campo investigativo.

En contraparte, los estudiantes identifican algunas actitudes negativas conductuales en los docentes del programa. Aldana y Joya (2011) aseguran que la dimensión conductual está representada por la disposición de los docentes a acciones relacionadas con la investigación. Las actitudes de la dimensión conductual se evidencian en su productividad investigativa Jiménez, 1993). En el programa, los estudiantes no conocen la producción académica de sus profesores; asimismo, consideran que algunos de ellos no motivan la construcción de proyectos innovadores, ya que estos les exigen capacitarse en temas que desconocen. Este último punto es importante para Zárate (2010), quien asevera que el enorme progreso en la construcción de conocimientos sobre diferentes temáticas es producto de la realización de investigaciones novedosas.

\section{Actitudes estudiantiles hacia la investigación formativa}

Aldana y Joya (2011) sugieren que las actitudes positivas de los educandos hacia la investigación surgen desde la dimensión conductual, en la cual los estudiantes demuestran la disposición que tienen para realizar procesos investigativos. Las actitudes positivas del estudiantado frente a la investigación formativa guardan cercana relación con el tema de investigación seleccionado. Por lo anterior, este se debe desarrollar teniendo en cuenta la temática con la cual el estudiante se sienta más motivado, de esta manera se obtendrán mejores resultados (Zapata, 2005).

Ahora bien, durante el ciclo de fundamentación y el ciclo profesional, en la mayoría de los casos, los estudiantes no tienen actitudes positivas frente a la investigación formativa, en parte debido a que no se sienten motivados por el personal docente. Cervantes et al. (2009) aseveran que las actitudes infieren de manera determinante sobre las percepciones individuales; actitudes negativas de los profesores generan estas mismas actitudes en el estudiantado e influyen directamente en él a través de la interacción social.

Aldana y Joya (2011) plantean que las actitudes negativas que tienen los estudiantes frente a la investigación formativa son influenciadas por sus relaciones con los docentes. Las fuentes consultadas mencionan que se observa indiferencia en algunos estudiantes del programa; durante el Seminario de Investigación es notoria la dificultad que causa esta actitud en la construcción del proyecto. Las actitudes conductuales del estudiante tienen gran incidencia en el desarrollo del proceso investigativo: si estas son negativas, interfieren en sus actitudes afectivas y cognitivas hacia la investigación (Aldana y Joya, 2011).

Las actitudes negativas también se reflejan en el poco espíritu investigativo que tienen los estudiantes del programa. Es importante desarrollar este aspecto, ya que es determinante del grado de preparación que tiene el estudiante para proponer solución a problemáticas sociales (Andreiev, 1978). A veces el poco espíritu investigativo se debe a que los educandos se desmotivan al tener que realizar su trabajo de grado en los entornos de práctica asignados por la institución.

En cuanto a los espacios académicos, algunos estudiantes concuerdan en que Comunicación Oral y Escrita, Epistemología de la Investigación y Metodología de la Investigación en el ciclo de fundamentación generan actitudes negativas. Las asignaturas de investigación en este ciclo no contribuyen al desarrollo de actitudes positivas, a causa de un discurso descontextualizado; gran parte del estudiantado tiene una percepción neutra o negativa frente a estos espacios. Rojas (2010), atribuye lo anterior al excesivo formalismo de algunos docentes frente a los procesos investigativos. En estos espacios académicos del campo investigativo, los docentes no siempre aplican estrategias adecuadas para la construcción de actitudes positivas y se muestran distantes de la realidad investigativa de los educandos en esta área. Balbo (2011), asegura que

Los docentes parten de la idea que [sic] los estudiantes ya deben saber qué implica investigar e intervenir en grupos sociales, cómo hacerlo y cómo producir los documentos escritos que estas prácticas suponen, es por esto que deben valerse por sus propios medios para lograr el aprendizaje; esto quiere decir que las deficiencias en términos investigativos persisten, ya que no han logrado desarrollar en las etapas precedentes las competencias para abordar las investigaciones. (p. 14).

Aldana y Joya (2011) concluyen que el docente debe reflexionar sobre su intervención pedagógica y sobre 
sus actitudes, ya que las actitudes dan lugar a sentimientos y pensamientos agradables sobre una temática, que en este caso es la investigación.

\section{Relaciones académicas}

Las actitudes positivas de docentes y estudiantes frente a la investigación formativa garantizan buenas relaciones académicas entre ellos. En el Programa de Licenciatura en Educación Física las fuentes de información coinciden en que existe la confianza adecuada para desarrollar los procesos investigativos. Teniendo en cuenta la clasificación propuesta por Pianta (1994), la relación académica positiva que en mayor medida se presenta en el programa entre docentes de Seminario de Investigación y estudiantes es participación positiva (positively involved), pero en algunos casos se presenta también la relación denominada promedio/funcional (average-functional). Según este autor, la primera relación se caracteriza porque docentes y estudiantes tienen buena comunicación y buena convivencia, lo cual propicia un espacio de participación activa. En tanto que la relación promedio/funcional se caracteriza como aquella en la cual docentes y estudiantes actúan con una adecuada empatía, colaboración y participación, aunque en ella pueden llegar a presentarse conflictos académicos. En este sentido, los procesos de investigación formativa que presentan el segundo tipo de relación tienen un correcto desarrollo, debido a que la investigación requiere de participación, integración y discusión académica.

Que las relaciones académicas del Programa de Licenciatura en Educación Física se cataloguen como de participación positiva o promedio/funcional es conveniente. Según Pianta (2001), estas relaciones académicas favorecen la convivencia en las instituciones educativas y permite desarrollar competencias de manera más eficaz. Brophy-Herb, Lee, Nievar y Stollak (2007) concuerdan en que los docentes y estudiantes que tienen relaciones positivas facilitan la comunicación y la autonomía en estos últimos, de esta manera se contribuye al desarrollo de competencias sociales que posteriormente permitirán construir diferentes competencias. Además, el estudiantado presenta una mayor disposición en la construcción de competencias cuando los profesores promueven la comunicación en sus clases y demuestran empatía.
Pianta (1999), concluye que las relaciones académicas entre docentes y estudiantes varían dependiendo de factores individuales y contextuales, tales como modelos representacionales, proceso de interacción, contexto familiar y contexto educativo. Para los estudiantes del Programa de Licenciatura en Educación Física el contexto universitario no contribuye plenamente al desarrollo del proceso investigativo, por esta razón es importante que los docentes del programa sean cercanos a la realidad investigativa de los educandos y, a través de la interacción, logren generar actitudes positivas en los estudiantes hacia la temática investigativa.

El estudiantado del Programa de Licenciatura en Educación Física también señala que en ocasiones los docentes no son coherentes con las sugerencias planteadas en sus proyectos investigativos, así no perciben estas indicaciones como un elemento de apoyo y construcción a su proceso. Para Pianta (1994), el tipo de relación disfuncional se caracteriza por que el docente o el estudiante demuestra poco interés por los procesos educativos; los alumnos identifican esta relación en docentes del programa que no imparten cátedra de espacios académicos del área investigativa.

Las relaciones académicas categorizadas como disfuncionales influyen negativamente sobre los educandos y pueden transformarse en relaciones distantes o sin compromiso (uninvolved), que se caracterizan por el poco interés de docentes y estudiantes por desarrollar procesos educativos (Pianta, 1994). Oren (2007) sostiene que relaciones negativas entre docentes y estudiantes obstaculizan el proceso de aprendizaje. Pianta (1994) asegura que estudiantes con relaciones académicas disfuncionales y distantes tienen mayores dificultades en el desarrollo de competencias. Dicho esto, los educandos consideran que algunos docentes demuestran apatía durante la asesoría de sus trabajos investigativos, lo cual los desmotiva y propicia falta de interés en ellos por la construcción de competencias propias de los procesos investigativos.

\section{CONCLUSIONES}

Las actitudes positivas de los estudiantes del Programa de Licenciatura en Educación Física aumentan significativamente a partir del séptimo semestre, 
debido a que en estos semestres los profesores que orientan los espacios académicos del campo investigativo construyen estas actitudes con su ejemplo.

Los docentes del programa mayoritariamente presentan actitudes positivas frente a la investigación formativa. Es de gran importancia que los profesores que orientan espacios académicos del campo investigativo sean personas con experiencia en esta área, creativas y capaces de contextualizar sus conocimientos investigativos a la disciplina de la educación física. Por otra parte, existen pocos docentes en el programa que tienen actitudes de indiferencia, desmotivación y desinterés frente a esta área.

Los espacios académicos del campo investigativo que se encuentran en el ciclo de fundamentación generan actitudes conductuales negativas en el estudiantado, puesto que en ocasiones los docentes presentan un discurso descontextualizado. Se debe agregar que en estos semestres los estudiantes aún no logran comprender la importancia que tiene la investigación formativa en la profesión docente.

Aunque no siempre los estudiantes del programa tienen actitudes conductuales positivas frente al campo investigativo, el Programa de Licenciatura en Educación Física presenta un alto compromiso social. El área investigativa está orientada a responder a las necesidades educativas de los diferentes contextos, desde el punto de vista de la educación física. Esto demuestra que el estudiantado tiene actitudes afectivas positivas en investigación.

\section{REFERENCIAS}

Aldana, G. y Joya, N. (2011). Actitudes hacia la investigación cientifica en docentes de metodología de la investigación. Tabula Rasa, 14, 295-309.

Andreiev, V. (1978). Evaluación pedagógica de las habilidades investigativas de los alumnos de los grados superiores y de los estudiantes en las condiciones de la prorgramación heurística de la enseñanza. Educación Superior Contemporánea, 1(21).

Avendaño, W. y Parada, A. (2013). El currículo en la sociedad del conocimiento. Educación y Educadores, 16(1), 159-174.

Balbo, J. (2011). Formación en competencias investigativas, un nuevo reto de las universidades. Recuperado dehttp://www.ucv.ve/fileadmin/user_upload/vrac/ documentos/Curricular_Documentos/Evento/Ponencias/Balbo_josefina.pdf.

Banguero, C. (2008). Una experiencia de investigación formativa. Entramado, 4(2), 70-76.

Brophy-Herb, H., Lee, R., Nievar, M. y Stollak, G. (2007). Gary Stollak Preschoolers social competence: Relations to family characteristics, teacher behaviors and classrool climate. Journal of Applied Developmental Psychology, 28, 134-148.

Campos, N. (2003). El docente investigador: su génesis teórica y sus rasgos. Educación, 27(2), 39-42.

Carrillo, B. (2009). Importancia del currículum oculto en el proceso de enseñanza-aprendizaje. Innovacion y Experiencias Educativas, 14, 1-10.

Cervantes, R., Cappello, H. y Castro, R. (2009). Análisis de las actitudes docentes hacia la educación científica. Un estudio del programa de enseñanza de las ciencias aplicado en escuelas priamrias de Ciudad Victoria, Tamaulipas. Revista Internacional de Ciencias Sociales y Humanidades, 19(1), 9-26.

Díaz, Á. (2005). La educación en valores: vatares del currículum formal, oculto y los temas transversales. Revista Electrónica de Investigación Educativa, 8(1), 1-12.

Eisner, E. (1979). The educational imagination On the design and evaluation of school programs. Standford: Stanford University.

Escobar, J. y Cuervo, Á. (2008). Validez de contenido y juicio de expertos: una aproximación a su utilización. Avances en Medición, 6, 27-36.

Hamui, A. y Ruiz, M. (2013). Investigacion en educación médica. Universidad Nacional Autónoma de México, 2(5), 55-60.

Hernández, I. (2009). El docente investigador en la formación de profesionales. Revista Virtual Universidad Católica del Norte, 27, 1-21.

Hildebrand, D. (2004). La importancia del respeto en la educación. Educación y Educadores, 7, 221-228.

Jiménez, B. (1993). Productividad en investigación del docente universitario. Espacios, 14(3).

Kirk, D. (1990). Educación física y currículum. Valencia: Universitat de Valéncia.

Magendzo, A., Abraham, M. y Dueñas, C. (1993). Manual para profesores Currículum y Derechos Humanos. Chile: Instituto Interamericano de Derechos Humanos. 
Malagón, L. (2008). El currículo: perspectivas para su interpretación. Investigación y Educación en Enfermería, 26(2), 136-142.

Miyahira, J. (2009). La investigación formativa y la formacion para la investigación en el pregrado. Revista Herediana, 20(3), 119-122.

Núñez Flores, M. y Vega Calero, L. (2011). La formacion investigativa y la tesis de pregrado para obtener la licenciatura en educación. Investigacion Educativa, 15(28), 32-40.

Oren, M. (2007). Child temperament, gender, teacher-child relationship, and teacher-child interactions. Humanities and Social Sciences, 67(8).

Parra, C. (2004). Apuntes sobre la investigación formativa. Educación y Educadores, 7, 57-77.

Pianta, R. (1994). Patterns of relationships between children and kindergarten teachers. Journal of School Psychology, 32(1), 15-32.

Pianta, R. (1999). Enhancing relationships between children and teachers. Washington, D. C., Londres: American Psychological Association.
Pianta, R. (2001). STRS. Studen-Teacher Relationships Scale. Florida: Psychological Assessment Resources.

Pirela, L. y Prieto, L. (2006). Perfil de competencias del docente en la función de investigador y su relación con la producción intelectual. Opción, 22(50), 159-177.

Robles, B. (2011). La entrevista en profundidad: una técnica útil dentro del campo antropofísico. Cuicuilco, 18(52), 39-49.

Rodríguez, F. (2012). Proceso de Bolonia (v): el currículo oculto. EducMed, 15(1), 13-22.

Rojas Betancur, M. (2010). La actitud estudiantil sobre la investigación en la universidad. Investigación \& Desarrollo, 18(2), 370-389.

Sánchez, R. y Meza, D. (2007). El currículo por competencias. Comunidad y Salud, 5(1), 1-3.

Zapata, O. (2005). ¿Cómo encontrar un tema y construir un tema de investigación? Innovación Educativa, 5(29), $37-45$.

Zárate, E. (2010). Innovación en la investigación. Peru Med, 27(3), 432-437. 